\title{
Effects of the Installation Angle of Raised Pavement Markers on a Horizontal Curve Section on the Line of Sight Induction Performance
}

\author{
Guo-hua Liang $\mathbb{D}^{1}{ }^{1}$ Dong Zhang $\mathbb{D}^{1},{ }^{1}$ Shi-bei Du, ${ }^{1}$ Yu-jie Yin, ${ }^{1}$ Rui Li, ${ }^{1}$ and Bo-rui Shi ${ }^{2}$ \\ ${ }^{1}$ School of Highway, Chang'an University, Xian, Shaanxi 710064, China \\ ${ }^{2}$ School of Traffic, Northeast Forestry University, Harbin, Heilongjiang 150040, China \\ Correspondence should be addressed to Dong Zhang; 294856372@qq.com
}

Received 14 May 2018; Accepted 4 July 2018; Published 17 July 2018

Academic Editor: Elio Masciari

Copyright ( $\odot 2018$ Guo-hua Liang et al. This is an open access article distributed under the Creative Commons Attribution License, which permits unrestricted use, distribution, and reproduction in any medium, provided the original work is properly cited.

\begin{abstract}
Different installation angles of raised pavement markers (RPMs) correspond to different incident angles of light, which affects the retroreflective performance of the RPMs. This paper explores how adjustments to the installation angle of the RPMs on a horizontal curve section affect a driver's line of sight induction. The conditions under which RPMs achieve the optimal effect of retroreflection are confirmed, and a driver's visual range at night is analyzed. The incident angle of light and the installation angle of RPMs are calculated when the optimal effect of retroreflection is achieved and the RPMs are installed along the radial direction (normal conditions). The differences in incident angles are compared. The results show that the differences in the incident angle under the two installment modes decrease as the radius increases, with a maximum value of 5.9 degrees. The improvement of the retroreflection with variations in the incident angle is analyzed using the STT-201A RPM measuring instrument. When the incident angle is decreased by 5.9 degrees and the observed angle is less than 2 degrees, the average increase in the coefficient of the retroreflection is only $12.6 \%$, indicating that the improvement of the retroreflection is not obvious. Therefore, a driver's perception of road alignment cannot be significantly improved by adjusting the installation angle of RPMs. Considering the complexity of the construction of a project, the installation angle should not be changed under normal conditions; namely, the RPMs should still be installed along the radial direction.
\end{abstract}

\section{Introduction}

Raised pavement markers (RPMs) are spikes fixed on road pavement that are used to divide lanes, mark lane edges, and effectively ease drivers' visual fatigue from the vibration warning produced by a vehicle when it rolls over them [1]. These devices help to reduce the probability of traffic accidents. Moreover, the reflective characteristic of RPMs can improve the road visibility at night and provide linear induction for drivers [2]. Due to their low in cost and easy installation, RPMs are widely used in road safety facilities. RPMs are shown in Figure 1.

Previous studies have mainly focused on where to set RPMs from the perspective of safety factors. Setting RPMs along a horizontal curve can improve drivers' curvature perception and greatly reduce the number of vehicles that veer out of their travel lane [3]. Velocity and deviation data of vehicles driving along curves have been analyzed, with the results indicating that the driving track of the driver differs from the line shape of the horizontal curves. The rate of change in the vehicle speed can be significantly reduced by installing RPMs, which is beneficial from a traffic safety perspective [4]. The induction effect of RPMs is the greatest at night, with collisions between vehicles being effectively reduced [5]. Moreover, there is a potential relationship between RPMs and pavement markings. Research results have shown that installing RPMs can effectively reduce the reflective requirements of pavement markings, which prolongs the service life of the markings [6].

Due to the increasing number of traffic incidents, studies have begun considering the layout of RPMs. In the United States, the recommended interval value for RPMs is as 


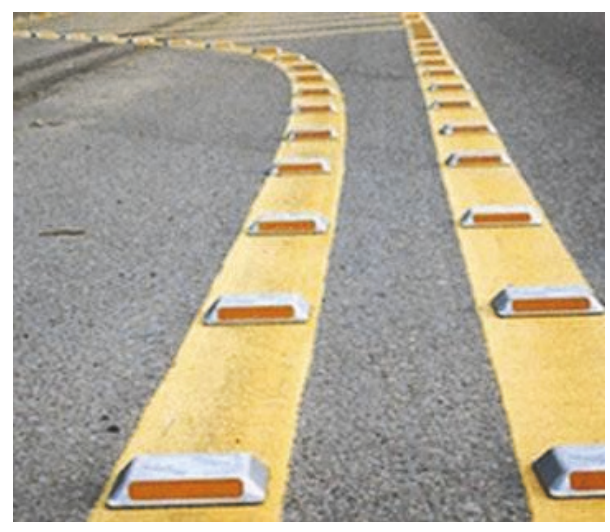

FIGURE 1: Raised pavement markers.

follows. The normal spacing is $\mathrm{N}(12.3 \mathrm{~m})$. However, on an expressway, the spacing should be $2 \mathrm{~N}-3 \mathrm{~N}$. When there is a single displaced solid line, the spacing should be less than $\mathrm{N} / 4$, and for a virtual solid line the spacing should be less than N/8 [7]. New Zealand regulated the interval value for RPMs according to different line types as follows. When RPMs are used on centerlines, lane lines, and no-overtaking lines, the setting interval is $10 \mathrm{~m}$. When RPMs are used on edge lines, the setting interval is $20 \mathrm{~m}$ [8]. Australia regulation call for an RPM setting interval of $24 \mathrm{~m}$ on roads with no illumination and $12 \mathrm{~m}$ in other cases [9]. In China, the recommended range of the location and spacing of RPMs for different situations is provided in the relevant specifications. When RPMs are used with pavement markings, the active luminescent type or directional reflective type should be employed. The setting interval of RPMs is 6 to $15 \mathrm{~m}$ and can be adjusted according to the actual situation [10].

Based on the above standards, several scholars have further studied the setting interval of RPMs. The impact of different setting intervals on traffic safety was determined by evaluating different RPM settings [11]. The initial setting interval of RPMs was determined and amended after the visual warning function was analyzed [12]. The setting interval of the RPMs and their matching form with pavement markings were considered to understand the role of RPMs in traffic safety [13]. However, previous papers have mainly focused on the safety and setting interval of RPMs. Few studies have focused on the installation angles of RPMs along horizontal curves, and there are few studies on RPMs in general. RPMs are usually installed along the edges of lines, lane lines, or centerlines, with most installed in the radial direction. As shown in Figure 2, RPMs can provide linear induction for drivers under poor lighting conditions at night. However, it is unclear whether different installation angles would affect a driver's perception of the road alignment due to the retroreflective characteristics of RPMs.

In this paper, the conditions under which RPMs achieve the optimal retroreflection effect were determined. Then, the installation angle of the RPMs and the incident angle of light were calculated. The relative improvement effect of the retroreflection compared with normal conditions was studied, and the relationship between the installation angle

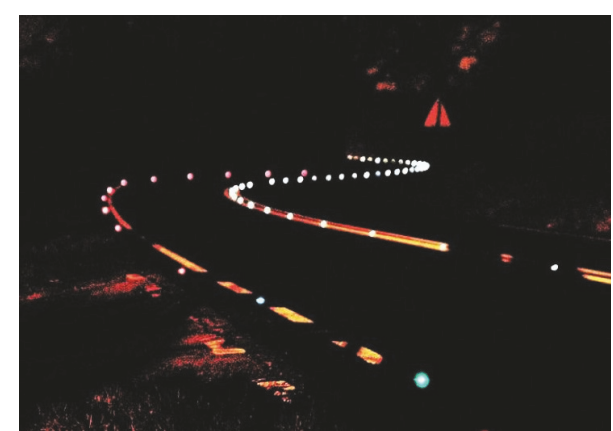

FIGURE 2: Effect of linear induction of RPMs at night.

of the RPMs and the line of sight induction at night was determined.

\section{Methodology}

\subsection{Study of the Optimal Retroreflection Effect Based on the Retroreflective Characteristics}

2.1.1. Retroreflective Characteristics of RPMs. Retroreflection is a characteristic where reflected light closely returns in the direction opposite to the incident light [14]. Coefficients of retroreflection are used to measure the retroreflective performance, with higher coefficients of retroreflection indicating better retroreflective performance. The coefficients of retroreflection vary depending on the different incident angles and observed angles, which affects the retroreflection performance.

According to the definition of Road Traffic Reflective Film (GB / T 18833-2012), the illumination axis is the ray emitted from the center of the reflex body that passes through the point of the light source, and the retroreflection axis is a specific ray emitted from the center of the retroreflector. The incident angle refers to the angle between the illumination axis and retroreflection axis, which is the angle between the vehicle light and the central axis of the reflector surface of the RPM. The observed angle refers to the angle between the illumination axis and observation axis, which is the angle between the vehicle light and the light reflected into the eyes of the driver. The RPM is smaller than the vehicle and the road. Therefore, the observed angle formed by the vehicle light and reflected light can be considered approximately unchanged with variations in the RPM installation angle.

\subsubsection{Conditions for Achieving the Optimal Retroreflection} Effect. As shown in Figure 3, $A B$ is the height of the vehicle light, $A O$ is the incident light ray, $D F$ is the line at the bottom edge of the reflector, and $O$ is the location of the center of the RPM. The observed angle is approximately unchanged from the above definition. Thus, when the incident light $A O$ is invariant, the RPM rotates around its central axis, and the central axis $O E$ of the RPM also rotates along with it. This forms a geometry that is shaped such as a circular truncated cone, where $C$ is the center of the upper surface. When $O E$ and $A O$ are in the same vertical plane, the reflector area 


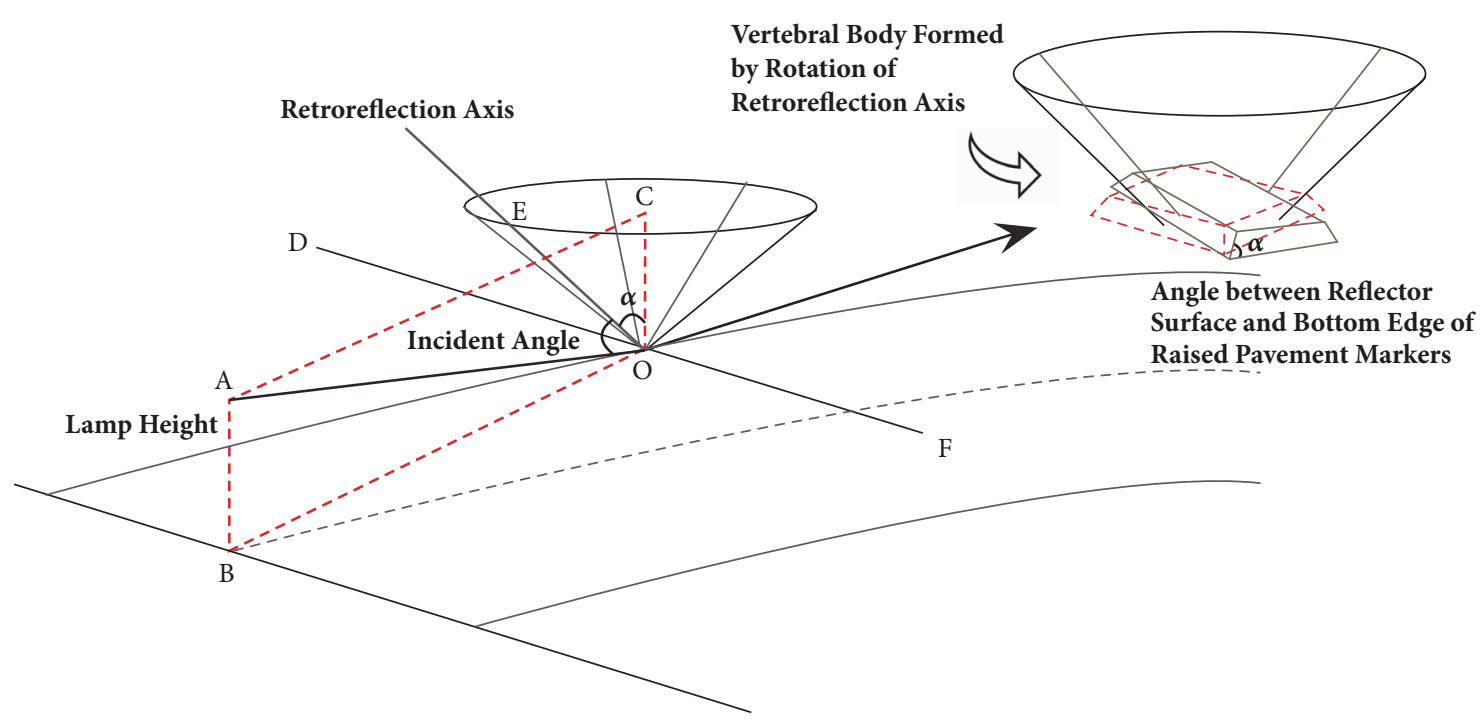

FIGURE 3: Change in the incidence angles of light.

is the largest in the driver's view, which indicates that the retroreflective effect of the RPM is optimal. At this point, the incident angle is the smallest, and it can be proven by simple geometric principles that the line DF located at the bottom edge of the RPM reflector is perpendicular to the plane where $A B O C$ is located. Therefore, $D F$ is perpendicular to $A O$. In other words, the RPM achieves the optimal retroreflection effect when the incident light is perpendicular to the bottom edge of the RPM reflector.

\subsection{Calculation of the Light Incident Angles and RPM Instal-} lation Angles Based on Visual Characteristics. Since there are a number of RPMs that can be seen in the driver's visual field of view during actual driving, the object of study must be determined before calculating the angles. Lin [15] simulated a night driving environment using the 3D-MAX software such that participants could perceive the line of sight induction effect achieved with different numbers of RPMs. The experimental results demonstrated that a better curvature perception was achieved when there are six RPMs in the driver's visual field. Therefore, this paper selects the six RPMs nearest the vehicle as the objects of study to calculate their installation angles and incident angles of light. The RPMs are denoted as Nos. 1-6.

Different RPMs have different physical dimensions, which will affect the calculation results. Raised Pavement Marker (GB T/24725-2009) stipulates that the angles between the reflector surface of RPMs and the ground should not exceed 65 degrees. In addition, to ensure visibility at night or in low-light conditions, traffic signs must comply with the minimum return rate standard specified in the Manual for Uniform Traffic Control Equipment (MUTCD) [16]. Therefore, the RPMs produced by $3 \mathrm{M}$ were selected as the object of study. The angle between the reflector surface and the ground was 37 degrees, and the area of the reflector surface was 17.25 $\mathrm{cm}^{2}$.
This study involves two key angles: the installation angle of the RPM and the incident angle of light. The installation angle is defined as the angle between the bottom edge of the RPM and the tangent line of the road, which is shown in Figure $5(\angle \theta)$. The incident angle is defined as the angle between the vehicle light and the central axis of the reflector surface of the RPM, which is shown in Figure $3(\angle A O E)$. Different installation angles will change the direction of the retroreflection axis on the reflective surface of the RPM, which will produce different incident angles, affect the retroreflective performance of the RPM, and affect the induction effect. To study whether the retroreflective performance of RPMs is improved with variations in the installation angle, the incident angle of light in two cases must be investigated, namely, for RPMs installed along the radial direction and for RPMs achieving the optimal retroreflection effect. Moreover, the RPM installation angle at which the optimal retroreflection effect is achieved was calculated.

2.2.1. Calculation of the Installation Angles and Incident Angles under the Optimal Retroreflection Effect. As shown in Figure 5, OF is the projection of incident light on the ground (it is approximately seen as the incident light), and it is perpendicular to the line $G H$ at the bottom of the reflector surface of the RPM, namely, $O F \perp G H$. Because the radius $F M$ is perpendicular to the tangent line of the road, $\angle \theta+\angle \beta=$ $\angle O F M+\angle \beta=90^{\circ}$. The installation angle $(\angle \theta)$ is calculated as follows:

$$
\begin{aligned}
\theta & =\arccos \left(\frac{\overline{O F^{2}}+\overline{F M^{2}}-\overline{O M^{2}}}{2 \overline{O F} * \overline{F M}}\right)=\angle O F M \\
\overline{O F} & =\sqrt{\overline{F M}^{2}-\overline{O M}^{2}}
\end{aligned}
$$

As shown in Figure 3, AO is the incident light, and $\mathrm{OE}$ is the retroreflection axis. $\mathrm{AB}$ is the height of the vehicle light, 


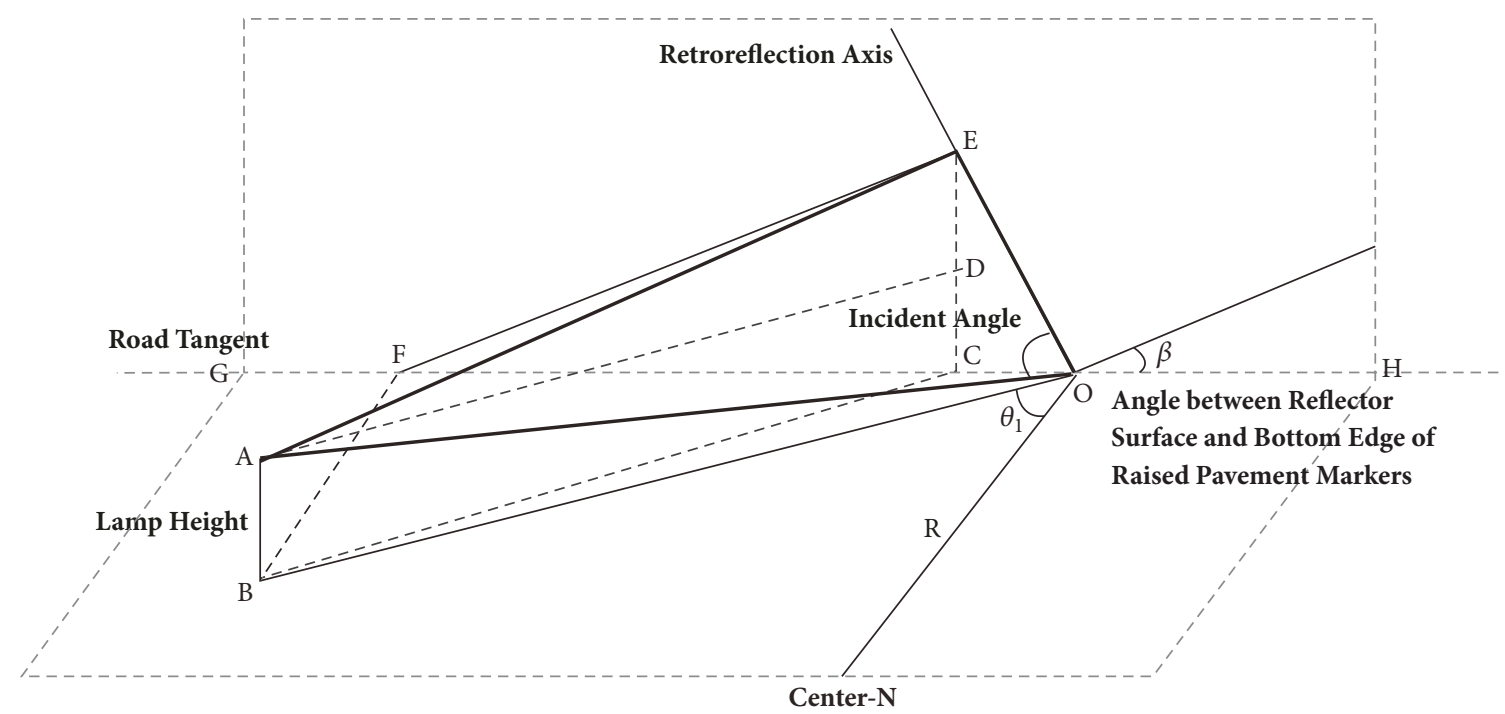

FIgURE 4: Simplified geometric diagram under normal conditions.

the calculated value of which is $0.6 \mathrm{~m}$. BO is the projection of the incident light on the ground, and its algorithm is the same as that of $\mathrm{OF}$ in formula (2). The quadrilateral ABOC is rectangular at the optimal retroreflection effect, and $\angle \alpha$ is equal to the angle between the reflector surface and the bottom edge of the RPM; namely, $\angle \alpha=37^{\circ}$ and $\angle B O E=$ $90^{\circ}-\angle \alpha=53^{\circ}$. The incident angle $(\angle A O E)$ is calculated as follows:

$$
\angle A O E=\angle B O E-\angle A O B
$$

2.2.2. Calculation of the Installation Angles and Incident Angles under Normal Conditions. Under normal conditions, the RPM is installed along the radial direction; then, the installation angle can be directly calculated as 90 degrees.

The incident angle $(\angle A O E)$ is simplified as shown in Figure 4 . The auxiliary line $B F$ is perpendicular to the road tangent line $G H$; the auxiliary line $F E$ is perpendicular to the retroreflection axis $O E$; the auxiliary line $E C$ is perpendicular to the road tangent line $G H$; the auxiliary line $A D$ is parallel to $B C . A B$ is the height of the vehicle light, $A O$ is the incident angle, and $O E$ is the retroreflection axis. $B O$ is the projection of the incident light on the ground, and its algorithm is the same as that in formula (2).

The incident angle $(\angle A O E)$ is calculated as follows:

$$
\angle A O E=\arccos \frac{\overline{A O}^{2}+\overline{E O}^{2}-\overline{A E}^{2}}{2 * \overline{A O} * \overline{E O}}
$$

The procedure for determining $A O, E O$, and $A E$ in formula (4) is as follows:

(1) Solve for the length of $A O$ :

$$
\overline{A O}=\sqrt{\overline{A B}^{2}+\overline{B O}^{2}}
$$

(2) Solve for the length of $E O$ :

$$
\begin{aligned}
& \overline{E O}=\overline{O F} * \cos \angle F O E \\
& \overline{O F}=\overline{B O} * \cos \angle B O F
\end{aligned}
$$

In the above formula, $\angle F O E=90^{\circ}-\angle \beta=53^{\circ}$ and $\angle B O F=90^{\circ}-\angle \theta_{1} . \angle \theta_{1}$ is equal to the $\angle O F M$ in Figure 5, and its value is the same as that of the optimal installation angle $(\angle \theta)$ calculated above.

(3) Solve for length of $A E$ (in the Rt $\triangle A D E$ ):

$$
\begin{aligned}
& \overline{A E}=\sqrt{\overline{E D}^{2}+\overline{A D}^{2}} \\
& \overline{E D}=\overline{E C}-\overline{C D}=\overline{E C}-\overline{A B}
\end{aligned}
$$

Because $\overline{E C} \perp \overline{O F}$,

$$
\begin{aligned}
& \overline{E C}=\frac{\overline{E F} * \overline{E O}}{\overline{O F}} \\
& \overline{A D}=\overline{B C}=\sqrt{\overline{B F}^{2}+\overline{F C}^{2}}=\sqrt{\overline{B F}^{2}+(\overline{F O}-\overline{C O})^{2}}
\end{aligned}
$$

According to the above formulas, different setting intervals of RPMs will affect the calculation parameters. By determining the visible arc length of drivers at night, the setting intervals of RPMs for different radii can be calculated.

\subsection{Determination of the Setting Interval of RPMs}

2.3.1. Analysis of the Visibility Range of Drivers at Night. Driving behavior is complex under actual conditions. Considering the feasibility and simplicity of the calculation, the following assumptions are made for the model of a driver's nighttime visual field of view.

(1) The vehicle always changes at a constant angle through the horizontal curve section, and the vehicle always drives on the centerline of the lane. 
TABLE 1: Corresponding arc length for each radius.

\begin{tabular}{lcccc}
\hline Radius $(\mathrm{m})$ & OA $(\mathrm{m})$ & OB $(\mathrm{m})$ & AB $(\mathrm{m})$ & $\begin{array}{c}\text { Setting interval of } \\
\text { RPMs }(\mathrm{m})\end{array}$ \\
\hline 30 & 7.3416 & 12.3016 & 5.2360 & 0.8727 \\
65 & 9.6039 & 20.6648 & 11.3446 & 1.8908 \\
100 & 10.9190 & 28.0808 & 17.4533 & 2.9089 \\
200 & 12.9489 & 47.5419 & 34.9066 & 5.8178 \\
400 & 14.6649 & 84.1202 & 69.8132 & 11.6355 \\
700 & 15.7174 & 137.4661 & 122.1730 & 20.3622 \\
1000 & 16.2276 & 190.2697 & 174.5329 & 29.0888 \\
\hline
\end{tabular}

(2) The divergent angle of the headlamp corresponds with the angle of the driver's range of visibility at night, which is always 10 degrees on a horizontal curve [17].

The driver's range of visibility at night is shown in Figure 5, where $O A$ and $\mathrm{OB}$ are the left and right boundaries of the visual area, respectively. In the course of the calculation, the width of the lane $(w)$ is $3.75 \mathrm{~m}$, the width of the vehicle (i) is $1.98 \mathrm{~m}$, and the distance between the driver's eye and the left edge of the vehicle $(d)$ is $0.66 \mathrm{~m}$. Then, the distance between the driver's eye and the left edge of the lane line $(O E)$ is $(w-i) / 2+d$. The formula to calculate the arc length $A B$ of the visible area is as follows:

$$
\begin{aligned}
A B & =\frac{\angle A M B \times \pi \times R}{180^{\circ}} \\
\angle A M B & =\angle O M B-\angle O M A
\end{aligned}
$$

According to the assumption regarding the driver's nighttime visual field of view, $\angle A O M=95^{\circ}$ and $\angle C O M=85^{\circ}$. Then, $\angle O M B$ and $\angle O M A$ can be calculated using the law of cosines.

2.3.2. Determination of the RPM Setting Interval. According to formula (12), the arc length $\mathrm{AB}$ is related to the radius. Analyzing all radii is neither realistic nor necessary. Therefore, the minimum radius of a circular curve in the Design specification for highway alignment (JTG D20-2017) was selected to calculate $\mathrm{AB}$. Then, the setting interval of the six RPMs was determined. To ensure the accuracy of calculation, the intervals take 4 decimal places. The results are shown in Table 1.

\section{Results}

3.1. Calculated Installation Angles and Incident Angles. At different radii, this paper calculates the incident angle of light and the installation angle of RPMs when the optimal retroreflection effect is achieved and the RPMs are installed along the radial direction (normal conditions). The results are shown in Table 2.

Table 2 shows that when the radius is less than $400 \mathrm{~m}$, the optimal installation angle of each RPM increases along with increases in the radius. When the radius is larger than $400 \mathrm{~m}$, the optimal installation angle of each RPM remains nearly unchanged even though the radius is still changing.
TABLE 2: Corresponding installation angles and incident angles for

\begin{tabular}{|c|c|c|c|}
\hline RPM number & Radius (m) & $\begin{array}{c}\text { Installation angle } \\
\text { (Optimal/Normal) } \\
\text { (degrees) }\end{array}$ & $\begin{array}{c}\text { Incident angle } \\
\text { (Optimal/Normal) } \\
(\text { degrees })\end{array}$ \\
\hline \multirow{7}{*}{1} & 30 & 27.99/90.00 & $40.95 / 46.85$ \\
\hline & 65 & $49.58 / 90.00$ & $43.40 / 47.65$ \\
\hline & 100 & $61.01 / 90.00$ & $45.15 / 48.57$ \\
\hline & 200 & $74.24 / 90.00$ & $47.87 / 49.02$ \\
\hline & 400 & $81.59 / 90.00$ & $50.06 / 50.55$ \\
\hline & 700 & $81.92 / 90.00$ & $50.81 / 50.99$ \\
\hline & 1000 & $82.12 / 90.00$ & $51.32 / 51.42$ \\
\hline \multirow{7}{*}{2} & 30 & $46.08 / 90.00$ & $43.39 / 47.95$ \\
\hline & 65 & $65.88 / 90.00$ & $45.43 / 48.66$ \\
\hline & 100 & $73.35 / 90.00$ & $47.24 / 49.19$ \\
\hline & 200 & $80.74 / 90.00$ & $50.06 / 50.64$ \\
\hline & 400 & $84.53 / 90.00$ & $51.52 / 51.72$ \\
\hline & 700 & $85.16 / 90.00$ & $52.06 / 52.15$ \\
\hline & 1000 & $85.81 / 90.00$ & $52.31 / 52.38$ \\
\hline \multirow{7}{*}{3} & 30 & $56.31 / 90.00$ & $45.10 / 48.59$ \\
\hline & 65 & $72.10 / 90.00$ & $47.07 / 49.33$ \\
\hline & 100 & $77.39 / 90.00$ & $49.09 / 50.19$ \\
\hline & 200 & $82.42 / 90.00$ & $51.03 / 51.42$ \\
\hline & 400 & $84.96 / 90.00$ & $52.01 / 52.18$ \\
\hline & 700 & $85.35 / 90.00$ & $52.44 / 52.54$ \\
\hline & 1000 & $85.69 / 90.00$ & $52.61 / 52.69$ \\
\hline \multirow{7}{*}{4} & 30 & $62.26 / 90.00$ & $46.67 / 49.26$ \\
\hline & 65 & $75.00 / 90.00$ & $48.49 / 50.05$ \\
\hline & 100 & $79.05 / 90.00$ & $50.05 / 50.86$ \\
\hline & 200 & $82.86 / 90.00$ & $51.52 / 51.86$ \\
\hline & 400 & $84.76 / 90.00$ & $52.26 / 52.44$ \\
\hline & 700 & $85.12 / 90.00$ & $52.58 / 52.71$ \\
\hline & 1000 & $85.38 / 90.00$ & $52.70 / 52.81$ \\
\hline \multirow{7}{*}{5} & 30 & $65.89 / 90.00$ & $47.84 / 49.95$ \\
\hline & 65 & $76.46 / 90.00$ & $49.36 / 50.62$ \\
\hline & 100 & $79.73 / 90.00$ & $50.63 / 51.34$ \\
\hline & 200 & $82.79 / 90.00$ & $51.81 / 52.16$ \\
\hline & 400 & $84.31 / 90.00$ & $52.41 / 52.62$ \\
\hline & 700 & $84.96 / 90.00$ & $52.66 / 52.83$ \\
\hline & 1000 & $85.23 / 90.00$ & $52.76 / 52.91$ \\
\hline \multirow{7}{*}{6} & 30 & $68.19 / 90.00$ & $48.65 / 50.38$ \\
\hline & 65 & $77.17 / 90.00$ & $50.13 / 51.25$ \\
\hline & 100 & $79.91 / 90.00$ & $51.02 / 51.70$ \\
\hline & 200 & $82.46 / 90.00$ & $52.01 / 52.39$ \\
\hline & 400 & $83.73 / 90.00$ & $52.51 / 52.77$ \\
\hline & 700 & $84.18 / 90.00$ & $52.72 / 52.93$ \\
\hline & 1000 & $84.39 / 90.00$ & $52.80 / 53.00$ \\
\hline
\end{tabular}
different radii.

Therefore, for those roads with a curve radius larger than 400 $\mathrm{m}$, the RPM installation angle is not related to the radius at 


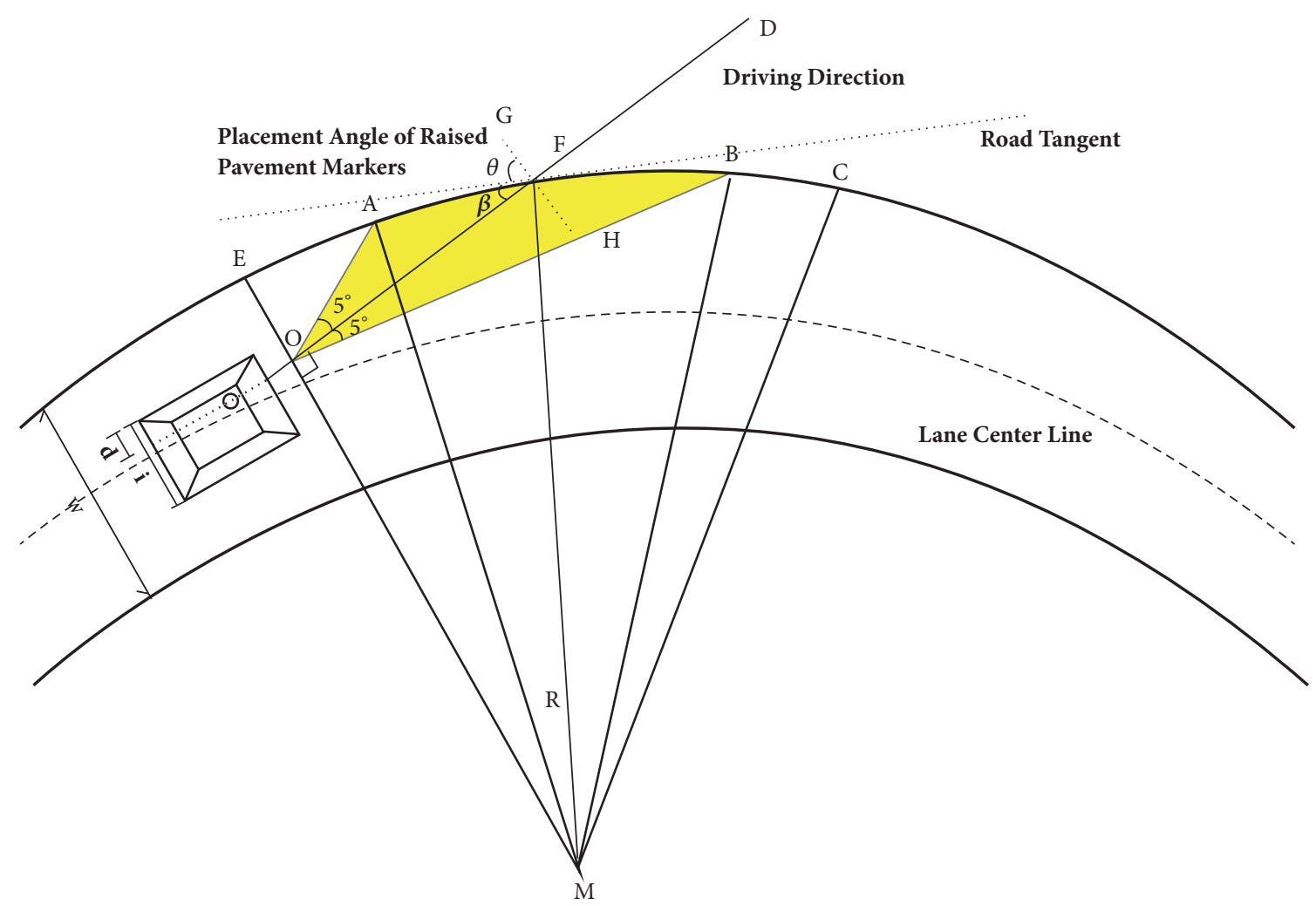

FIGURE 5: Visible area of drivers.

which the optimal retroreflection effect is achieved. Namely, the same installation angle can be used on different curve radii.

When the radius is less than $400 \mathrm{~m}$, the incident angles under two installation modes increase with increases in the radius. When the radius is larger than $400 \mathrm{~m}$, the incident angle under two installation modes tends to be stable and no longer increases with the radius.

3.2. Changes in the Difference between the Incident Angles. The difference between the incident angles of the RPMs installed along the radial direction and the RPMs rotated to achieve the optimal retroreflection effect is shown in Figure 6.

The difference between the incident angles of the RPMs installed along the radial direction and the RPMs rotated to achieve the optimal retroreflection effect decreases with increases in the radius. When the radius is larger than 400 $\mathrm{m}$, the difference between the incident angles of the two cases remains nearly unchanged with changes in the radius, and the average differences of Nos. 1-6 are 0.51 degrees, 0.17 degrees, 0.12 degrees, 0.11 degrees, 0.12 degrees, and 0.13 degrees, respectively. In other words, the incident angle when the RPM is installed along the radial direction nearly approaching the incident angle at which the optimal retroreflection effect is achieved. When the radius is less than $400 \mathrm{~m}$, the difference between the incident angles of the two cases is large. In particular, when the radius is $30 \mathrm{~m}$, the difference in the incident angle of the No. 1 RPM is the maximum, with a value of 5.9 degrees.

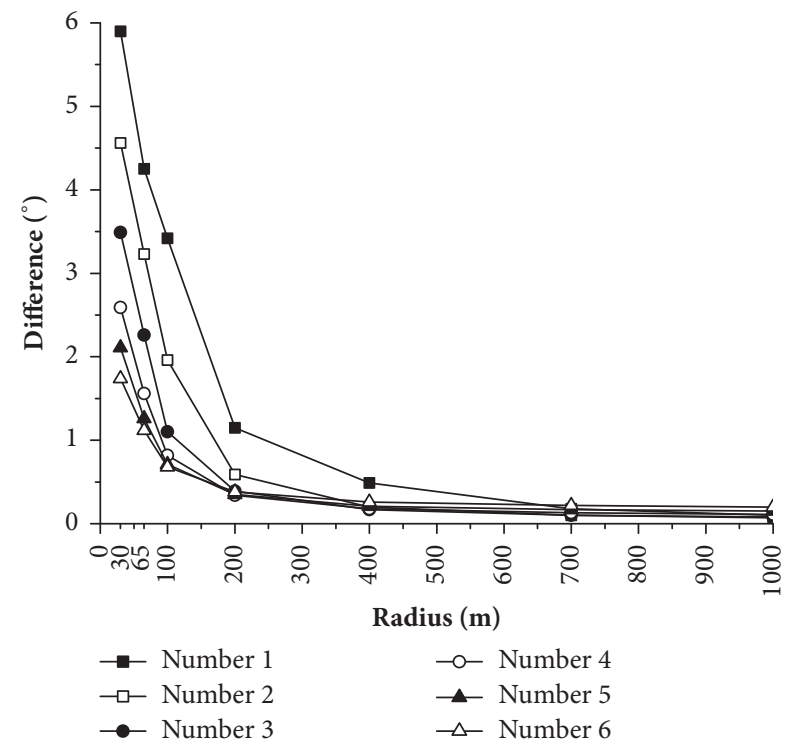

FIGURE 6: Change in the difference between the incident angles.

\section{Discussion}

4.1. Analysis on the Necessity of Changing the Installation Angle. To further understand the improvement in retroreflection caused by the change in the incident angle, we measured the coefficient of luminous intensity of RPMs using an STT-201A type measuring instrument indoors (Tianjin, 
China). The incident angle range was 0 degrees \pm 20 degrees (taking \pm 5 degrees as a scale unit). The observed angle used to determine the basic value of the coefficient of the luminous intensity in Raised pavement markers (GB/T 247252009) was chosen as the observed angle for this experiment. In this experiment, we recorded the coefficient of luminous intensity every 5 degrees, with a reduction from 20 degrees to 0 degrees, and the corresponding coefficient of retroreflection was calculated according to formula (14). A larger retroreflection coefficient indicated a better effect.

$$
R_{A}=\frac{R_{I}}{A}
$$

where $R_{A}$ is the coefficient of retroreflection, $R_{I}$ is the coefficient of luminous intensity, and $\mathrm{A}$ is the area of the reflector surface of the sample, which is $17.25 \mathrm{~cm}^{2}$.

The coefficients of luminous intensity and the coefficients of retroreflection of the RPMs under laboratory conditions are shown in Table 3.

Table 3 shows that when the observed angles are the same, the coefficients of retroreflection $\mathrm{R}_{\mathrm{A}}$ increase when the incident angle decreases by 5 degrees, and the effect of retroreflection is improved to some extent. When the observed angle is less than 2 degrees, the average improvement in $\mathrm{R}_{\mathrm{A}}$ is only $10.48 \%$, and the retroreflection improvement effect is not obvious. When the observed angle is 2 degrees, the maximum value of the improvement in $\mathrm{R}_{\mathrm{A}}$ is only $4 \mathrm{mcd} / \mathrm{lx} / \mathrm{m}^{2}$, and the retroreflection improvement effect is weak.

Therefore, this paper analyzes the retroreflection improvement effect corresponding to the maximum difference (5.9 degrees) of the incident angle when the observed angle is less than 2 degrees. From the data in Table 3, we can calculate the average increase in $\mathrm{R}_{\mathrm{A}}$ when the incident angle decreases by 10 degrees (decreasing from 20 degrees). Moreover, because there is a linear relationship between $\mathrm{R}_{\mathrm{A}}$ and a given observed angle, interpolation is used to calculate the retroreflection improvement effect when the incident angle decreases by 5.9 degrees. The result shows that the average increase in $\mathrm{R}_{\mathrm{A}}$ is only $12.6 \%$, and the retroreflection improvement effect is minor.

The experiment was carried out under good conditions. Drivers would be disturbed by other factors when driving at night, and the actual improvement effects of retroreflection would be lower than those observed in this experiment. Therefore, even if the RPMs are rotated to achieve the optimal retroreflection effect, the effect of the sight induction is not significantly improved. Moreover, rotating the RPMs by a small angle to achieve the optimal retroreflection effect increases the complexity of engineering construction. Therefore, the installation angle of the RPMs should not be changed under normal situations, namely, the RPMs should still be installed along the radial direction.

4.2. Retroreflection Effect of RPMs at Clothoids. Based on the circular curve, the model of a driver's nighttime visual field of view was built, and the setting interval of RPM was calculated. However, the clothoid is an integral part of the horizontal curve. When the road alignment is complicated (e.g., a circular curve with different radii), a clothoid is essential. Therefore, the above conclusions cannot be applied to clothoid and thus need to be further discussed.

By selecting the minimum length of the clothoids specified in the Design specification for highway alignment (JTG D20-2017) and combining the above conclusion that a better curvature perception was achieved when there are six RPMs in the driver's visual field, the setting interval of RPMs at clothoids is calculated. The results are shown in Table 4 .

According to formula (3) and formula (4), the incident angles under two installation modes were calculated. The result shows that the difference of incident angle increases with the increase in the radius. However, the maximum value is only 2.5 degrees, and the average improvement in retroreflection is only 5.24\%. Compared with the circular curve, the retroreflection effect is weaker. Therefore, it is not recommended that the installation angle of RPMs be changed at clothoids; namely, the RPMs should still be installed along the radial direction.

\section{Conclusions}

In this paper, the relationship between the installation angle of RPMs and the effect of the sight induction, specifically, the formulas of the installation angle of RPMs and the light of incident angle, were derived. By building the model of a driver's nighttime visual field of view, the setting interval of RPMs at horizontal curves was determined. The results of angle value show that the maximum difference of incident angles at circular curves and clothoids is 5.9 degrees and 2.5 degrees, respectively, and that the average improvement of retroreflection is $12.60 \%$ and $5.24 \%$, respectively. The driver's perception of the road alignment cannot be significantly improved. Consequently, the RPMs at horizontal curves should maintain the existing installation angle.

The RPMs are sometimes set with the line markings, and the setting interval is adjusted according to the actual situation. This condition will have an impact on the result, so it should be taken into account in future studies. Moreover, considering the complexity and safety of construction, the test of coefficient of retroreflection is not measured outdoors. When conditions permit, tests can be carried out on the actual road.

\section{Data Availability}

The data used to support the findings of this study are available from the corresponding author upon request.

\section{Conflicts of Interest}

The authors declare that there are no conflicts of interest regarding the publication of this paper.

\section{Acknowledgments}

This work was supported by Fundamental Research Funds for the Central Universities of China (no. 310821172007, no. 


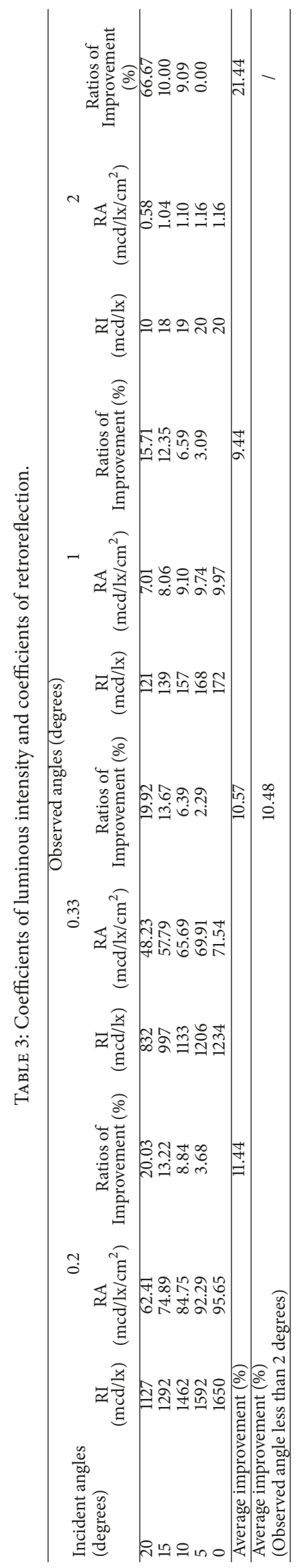


TABLE 4: The setting interval of RPMs at clothoids.

\begin{tabular}{lcc}
\hline Radius $(\mathrm{m})$ & $\begin{array}{c}\text { Minimum length of } \\
\text { clothoid }(\mathrm{m})\end{array}$ & $\begin{array}{c}\text { Setting interval of } \\
\text { RPMs }(\mathrm{m})\end{array}$ \\
\hline 30 & 20 & 3.3333 \\
65 & 25 & 4.1667 \\
100 & 35 & 5.8333 \\
200 & 50 & 8.3333 \\
400 & 70 & 11.6667 \\
700 & 85 & 14.1667 \\
1000 & 100 & 16.6667 \\
\hline
\end{tabular}

300102218410, no. 300102218521, and no. 300102218409) and the Shaanxi Provincial Science and Technological Project (no. 2017JM5104). The authors thank Yu Li for her contributions to this paper.

\section{References}

[1] P. Sherrill, G. W. Burkett, D. A. Bennett, and S. A. Velinsky, "Automated system for the replacement of raised pavement marker," Mechanics Based Design of Structures and Machines, vol. 40, no. 1, pp. 96-108, 2012.

[2] A. M. Pike, "Laboratory-Based Retroreflectivity Assessment of Raised Retroreflective Pavement Markers," Transportation Research Record, vol. 2612, pp. 113-120, 2017.

[3] J. L. Hammond and F. J. Wegmann, "Daytime effects of raised pavement markers on horizontal curves," ITE Journal (Institute of Transportation Engineers), vol. 71, no. 8, pp. 38-41, 2001.

[4] P. Zador, H. S. Stein, P. Wright, and J. Hall, "Effects of chevrons, post-mounted delineators, and raised pavement markers on driver behavior at roadway curves," Transportation Research Record, vol. 1114, pp. 1-10, 1987.

[5] P. H. Wright, P. L. Zador, C. Y. Park et al., "Effect of pavement markers on nighttime crashes in Georgia," in Insurance Institute for Highway Safety, Washington, DC, USA, 1982.

[6] H. T. Zwahlen and T. Schnell, "Minimum in-service retroreflectivity of pavement markings," Transportation Research Record, no. 1715, pp. 60-70, 2000.

[7] Federal Highway Administration, Manual on Uniform Traffic Control Devices for Streets and Highways, Washington, DC, USA, 2012

[8] NZ Transport Agency, Manual of Traffic Signs and Markings, New Zealand, 2010.

[9] Transport Road and Traffic Authority, Driver qualification handbook, Australia, 2010.

[10] Ministry of Transport of the People's Republic of China, Road traffic signs and markings, Beijing, China, 2009.

[11] B. Persaud, G. Bahar, C. J. Mollett, and C. Lyon, "Safety evaluation of permanent raised snow-plowable pavement markers," Transportation Research Record, no. 1897, pp. 148-155, 2004.

[12] X. Y. Wang, S. S. Ye, and L. Ge, "Analysis of the anticoagulant activity and ingredients of chives," Journal of Chinese Institute of Food Science and Technology, vol. 15, no. 3, pp. 216-222, 2015.

[13] Y. Jiang, "The Effectiveness and Criteria for Placement of Raised Pavement Markers (Synthesis Study)," Purdue University FHWA/IN/JTRP-2006/06, 2006.
[14] J. Guo, Y.-w. Guo, and J.-g. Pan, "A retroreflective BRDF model based on prismatic sheeting and microfacet theory," Graphical Models, vol. 96, pp. 38-46, 2018.

[15] G. B. Lin, Design of traffic safety facilities based on the drivers visual characteristics (masters thesis, Changan University, Xian, China, 2011.

[16] M. Khalilikhah and K. Heaslip, "The effects of damage on sign visibility: An assist in traffic sign replacement," Journal of Traffic and Transportation Engineering (English Edition), vol. 3, no. 6, pp. 571-581, 2016.

[17] Standardization Administration of the People's Republic of China, Motor vehicle headlamps equipped with filament lamps, Beijing, China, 2007, GB 4599-2007. 


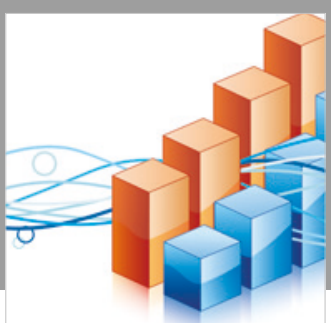

Advances in

Operations Research

\section{-n-m}
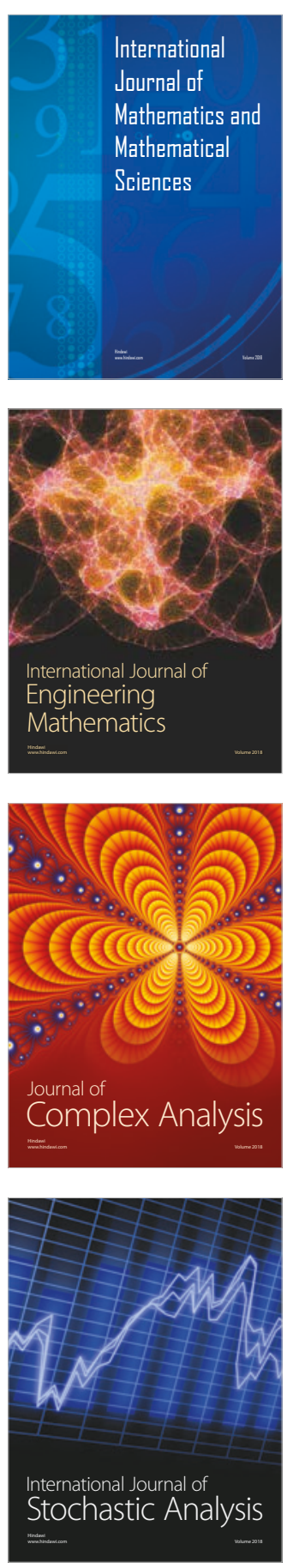
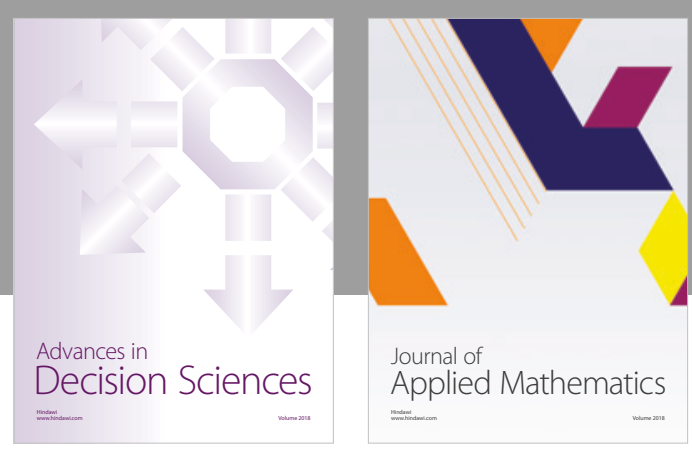

Journal of

Applied Mathematics
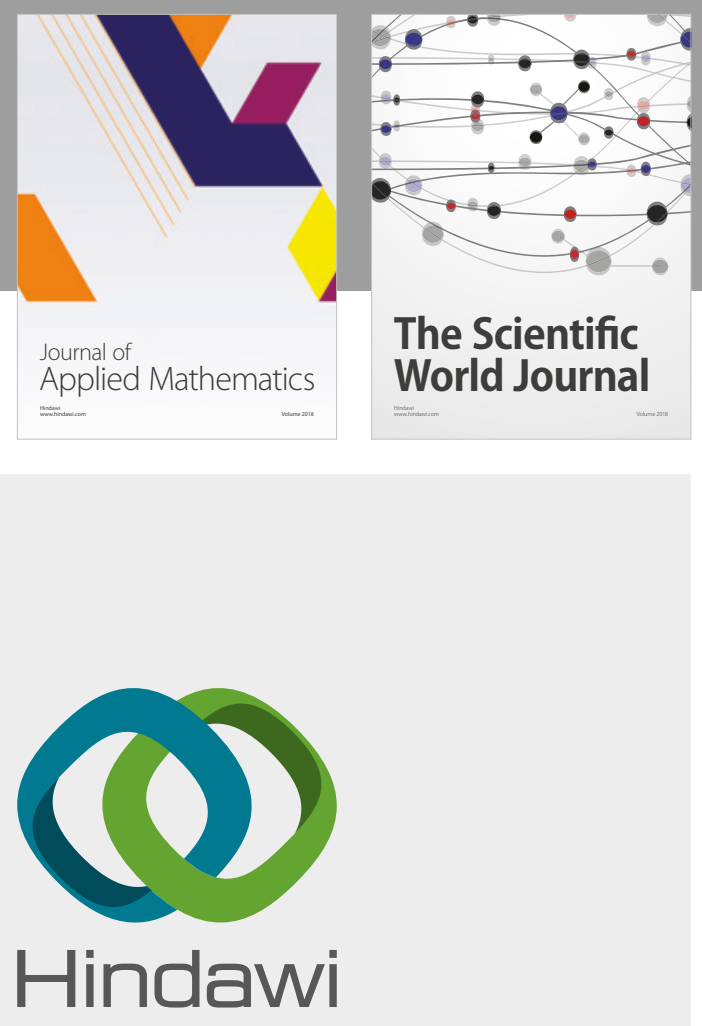

Submit your manuscripts at

www.hindawi.com

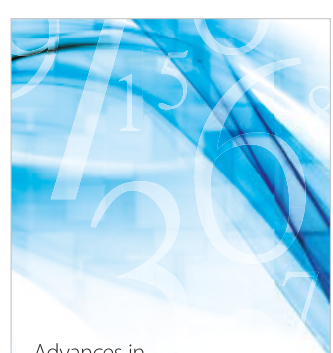

Advances in
Numerical Analysis
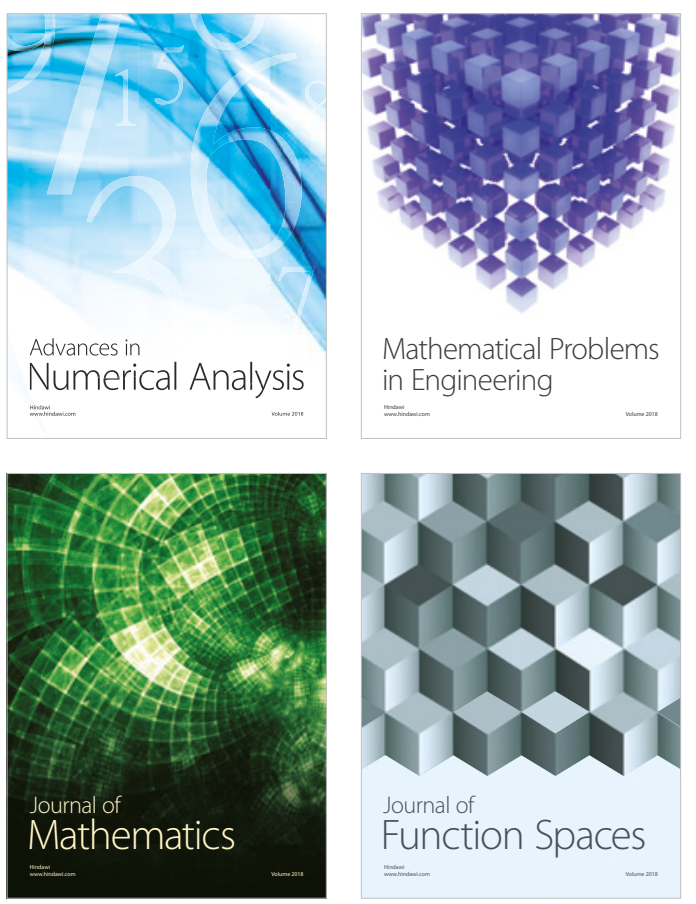

Mathematical Problems in Engineering

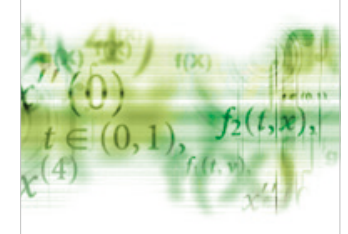

International Journal of

Differential Equations

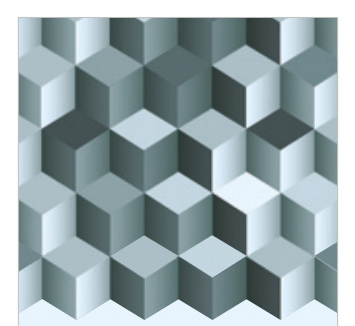

Journal of

Function Spaces

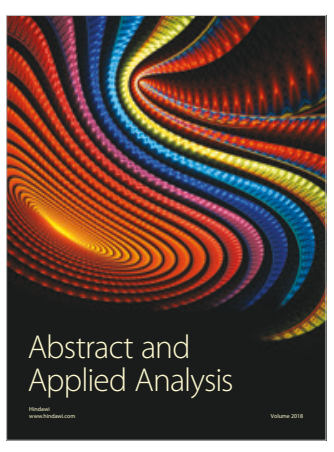

The Scientific

World Journal

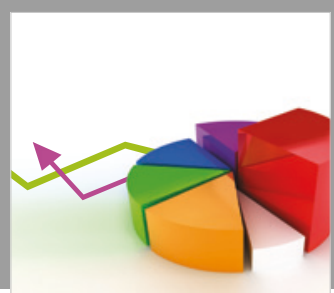

Journal of

Probability and Statistics
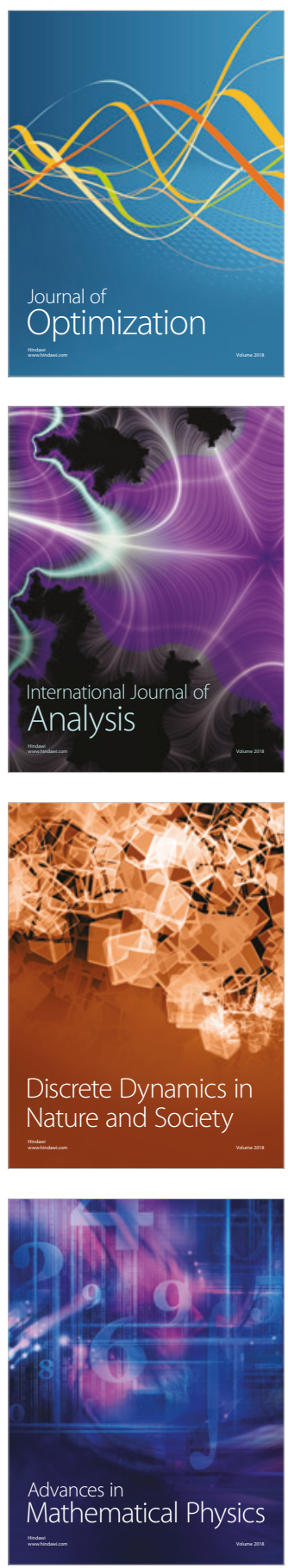